\title{
Fourier Transform Near Infrared Raman Spectroscopy in Studies on Connective Tissue
}

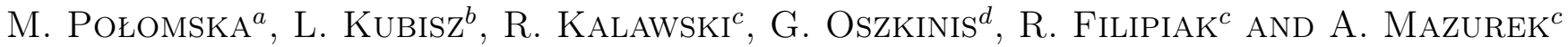 \\ ${ }^{a}$ Institute of Molecular Physics, Polish Academy of Sciences, M. Smoluchowskiego 17, 60179 Poznań, Poland \\ ${ }^{b}$ Department of Biophysics, Poznań University of Medical Sciences, Fredry 10, 61-701 Poznań, Poland \\ ${ }^{c}$ Department of Cardiac Surgery, Municipal Hospital, Szkolna 8/12, 61-417 Poznań, Poland \\ ${ }^{d}$ Department of General and Vascular Surgery, Poznań University of Medical Sciences, Poznań, Poland \\ Studies of tissue such as bones and veins often are carried out by means of microscopic and ultrasonographic \\ methods. Therefore studies of changes caused by pathological or physical factors often are limited to morphological \\ level. Application of Raman spectroscopy permits to associate morphological changes and molecular changes. \\ The paper presents preliminary studies of temperature and ionizing radiation effects on Raman spectra of bone \\ and collagen. Furthermore, effects of pathological factors on Raman spectra of human veins had been studied. \\ It was noticed that heating of bone collagen up to $110^{\circ} \mathrm{C}$ gives the reversible changes in spectra. In the case \\ of $\gamma$-irradiation, any effects were observed up to the dose of $1 \mathrm{MGy}$ on mineral component of bone, whereas \\ the influence on collagen was observed for doses higher than $100 \mathrm{kGy}$. The Raman spectra of varicose vs. nor- \\ mal veins permit to conclude some important changes observed for veins from chronic venous insufficiency patients.
}

PACS numbers: 78.30.-j, 78.30.Jw, 61.80.Ed, 61.82Pv, 81.05.-t, 87.-g, 87.93.-j, 87.64.K-

\section{Introduction}

Connective tissues are studied by means of different methods, among which promising method is Raman spectroscopy [1-8]. Collagen is one of the most important components of connective tissues such as tendons and bones. Collagen is the main protein making about $25 \%$ of the total protein content of human body. It is also contained in blood vessels. In bones, fibres of type I collagen constitutes about $85-90 \%$ of organic matrix, upon which the mineral crystals grew. The specific amino acid sequence of collagen makes triple helix, which is generally composed of the amino-acid sequence repeat (X-YGlycine) ${ }_{n}$, with proline and hydroxyproline often present at positions $\mathrm{X}$ and $\mathrm{Y}$. Another important biopolymer constituting blood vessel wall is elastin. It was shown that varicose veins contained less collagen and elastin in the comparison with normal veins [1]. Usually pathological and normal veins are studied by means of histological, electron microscopic and ultrasonographic studies $[2,3]$. Pathological and other changes caused by physical factors such as ionizing radiation and temperature, appearing in the studied material, may be related to changes in the structure of molecular group being basic component of proteins. These changes can be studied comparing the Raman spectra of native material and the material modified by biological and physical factors. In the last decade near infrared (NIR) Raman spectroscopy appeared to be an extremely useful method in studies on biomaterials. Using laser irradiation of $1064 \mathrm{~nm}$ we avoid fluorescence, the denaturation and dehydration along with thermal degradation of biomolecules [4-6]. The Fourier transform (FT) NIR Raman spectroscopy technique records frequencies associated with various vibrational motions between covalently bonded atoms. Raman peak position, full width at half maximum (FWHM), peak surface area permit to obtain information on the composition of the studied material. Raman spectroscopy offers the alternative analysis method instead of the common histopathological examination. Present work reports results of our Raman studies on effects of pathological and physical factors on the following tissues: vein vessel wall, bone and solid-state collagen.

\section{Material and method}

The measurements were performed on bones, Bovine Achilles Tendon (BAT) collagen type I, and vein wall samples. The BAT collagen was purchased in SIGMA Company.

Bone samples were obtained from the central part of the diaphyses of adult bovine femurs (2-3 year old animals). The bones were mechanically cleaned and washed in a $0.1 n$ solution of $\mathrm{NaCl}$ soon after the animals had been slaughtered. The samples were machine-cut using a diamond saw with continuous water irrigation to minimise thermal damage during the machining process. Next the samples were dried at $294 \mathrm{~K}$ in the air and irradiated using a ${ }^{60} \mathrm{Co}$ source. The samples were irradiated with the following doses: 10, 25, 50, 100, 500, 750 and $1000 \mathrm{kGy}$, in dry air, at room temperature and with the dose power of $1.5 \mathrm{kGy} / \mathrm{h}$.

Vein wall samples were taken from patients 50-60 years old. Veins applied in CABG (Coronary Aortic Bypass Grafts) operations were burden with any pathological changes, whereas veins taken from chronic venous insufficiency patients were pathologically changed. Samples were mechanically cleaned and washed in a $0.1 n$ solution of $\mathrm{NaCl}$, dried at room temperature to remove free water and submitted under experimental procedure. The study on human vein walls was agreed with the local Ethical Committee. 
The NIR Raman spectra of $\gamma$-irradiated bone and veins were measured at room temperature using Bruker IFS66 FRA 106 Raman spectrometer. The samples were excited with a continuous wave diode-pumped YAG:Nd laser operated at power of $\approx 100 \mathrm{~mW}$. The spectra were taken with 1000 scans and $4 \mathrm{~cm}^{-1}$ spectral resolution. Temperature Raman studies of BAT collagen were performed using Linkam cooling- heating stage.

\section{Results and discussion}

Figure 1 presents Raman spectra of bone, blood vessels and collagen. The bands related to molecular groups of different amino acids were found in all materials. Additionally, the bands related to mineral component assigned to hydroxyapatite (HAP) appeared in the Raman spectra of bone tissue. The bands positions and their tentative

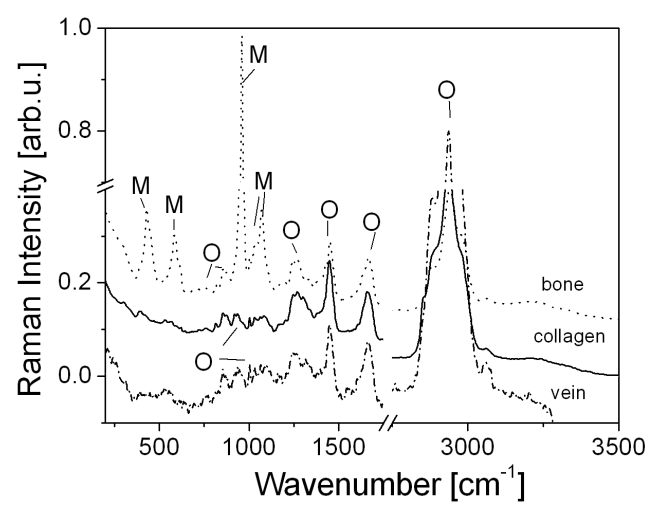

Fig. 1. Raman spectra of bone, vein and collagen measured at room temperature. $\mathrm{M}$ - mineral part, $\mathrm{O}-$ organic matrix.

assignments for bone, vein and collagen are presented in Table. The spectral assignment of Raman bands has been made using earlier studies on bones, collagen and other biomaterials [7-10]. The main molecular constituents of the studied tissues determine their the most important features. Therefore, the major bands in recorded Raman spectra are associated with the vibrational modes of collagen and elastin. The amide $\mathrm{I}$ at $\approx 1665 \mathrm{~cm}^{-1}$, the amide III at $\approx 1255 \mathrm{~cm}^{-1}$ and $\mathrm{CH}_{3}$ stretching symmetric vibrations at $2936 \mathrm{~cm}^{-1}$, which are sensitive to the secondary structure of protein were found in all spectra. Therefore, the Raman spectroscopy allowed to study individual components of bone as well as their interactions. As a result of the action of external factor and interactions between components of more complex materials, changes in Raman spectra were observed. Some lines disappeared, while other changed their intensity and position.

\subsection{Raman studies of $\gamma$-irradiated bone tissue}

$\gamma$-radiation is used in sterilization of medical products and food preservation, the dose used varies between 25

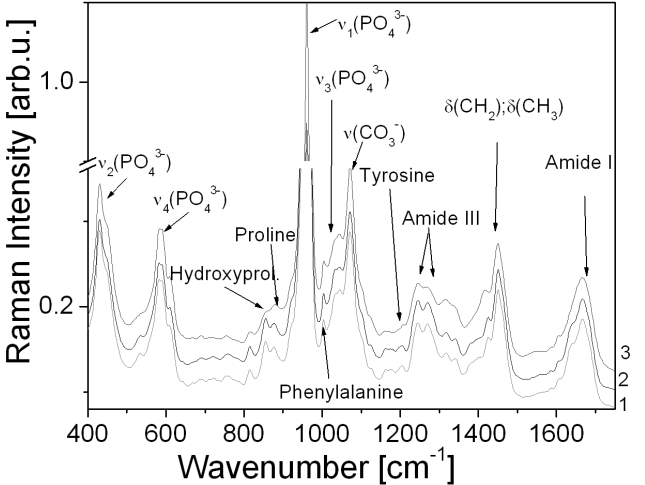

Fig. 2. Raman spectra of bone normal and $\gamma$ -irradiated. (1) - non-irradiated, (2) - $100 \mathrm{kGy,} \mathrm{(3)}$ $-1000 \mathrm{kGy}$.

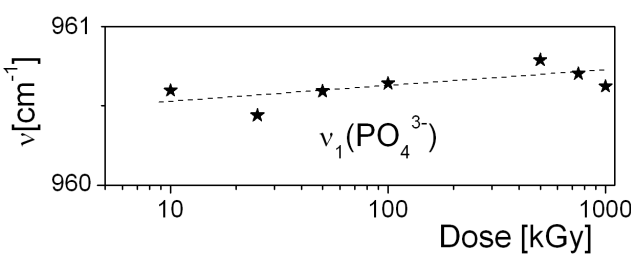

Fig. 3. The dose dependence of $\nu_{1} \mathrm{PO}_{4}^{3-}$ stretching vibration in bone.

and $50 \mathrm{kGy}$ [11]. In the case of bone, irradiation decreases the number of bacteria and does not change the structure of collagen even up to the dose of $100 \mathrm{kGy}$ [12]. Raman spectra of bone tissue for normal and $\gamma$-irradiated bone are presented in Fig. 2. Sensitivity of mineral (HAP) and organic components of bone to $\gamma$ radiation are dissimilar.

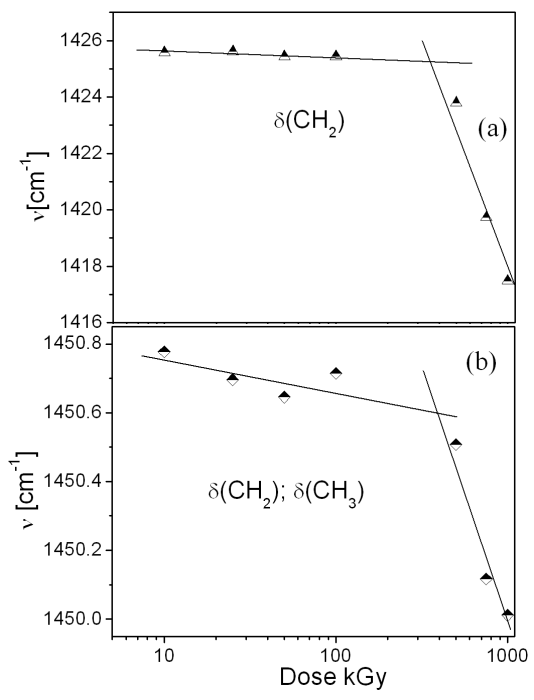

Fig. 4. The dose dependence of vibrations related to $\delta\left(\mathrm{NH}_{2}\right)$ (a) and to $\delta\left(\mathrm{NH}_{2}\right)$ and $\delta\left(\mathrm{NH}_{3}\right)$ vibrations of organic matrix in bone. 
TABLE

Raman bands positions in $\mathrm{cm}^{-1}$ and their assignment for collagen, bone and vein.

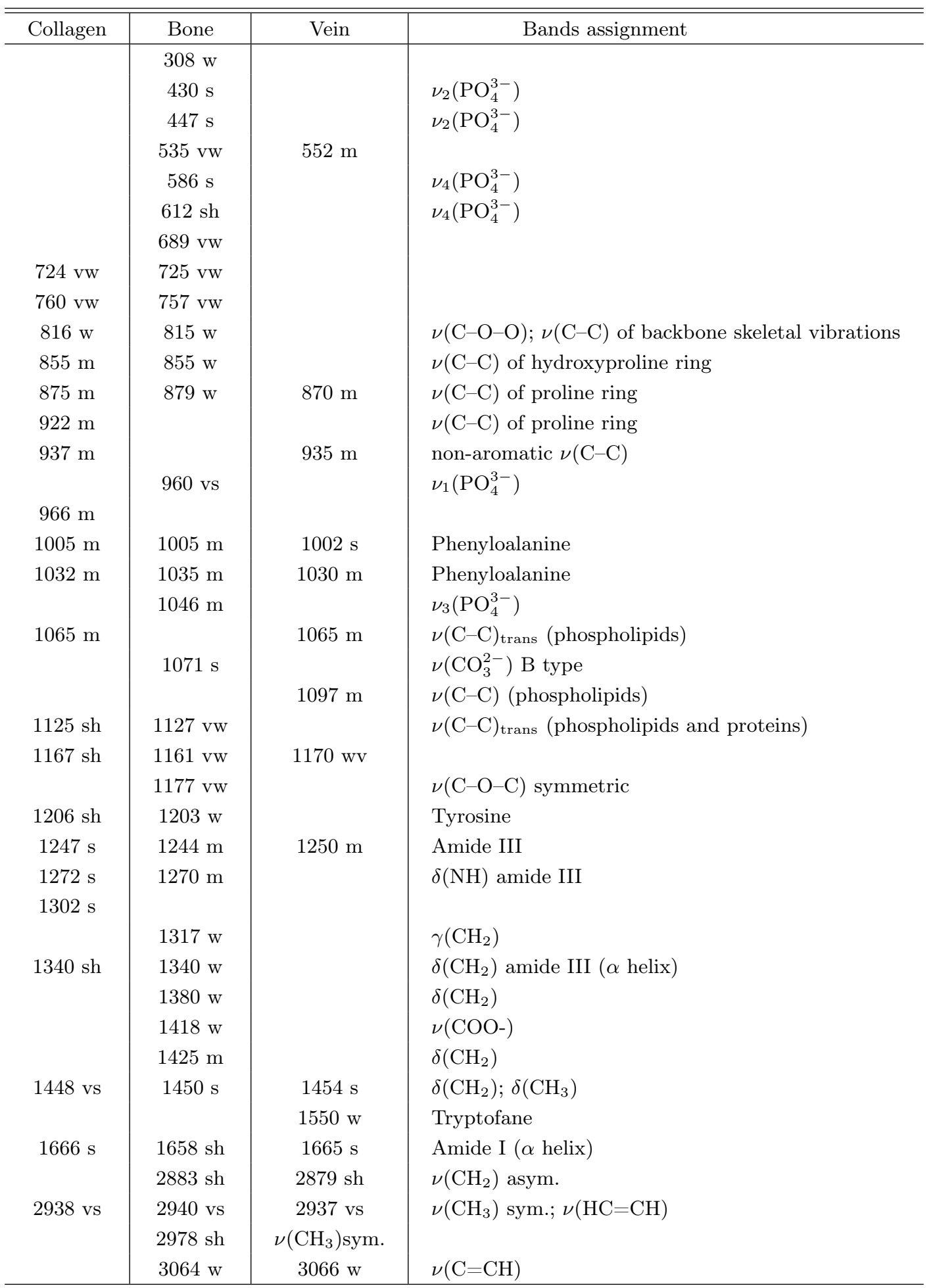

vs - very strong; s - strong; $\mathrm{m}$ - medium; w - weak; vw - very weak; sh — shoulder; $\nu$ - stretching; $\delta$ - deformation; $\gamma$ - twisting

As the example of the dependency of the Raman band position on the $\gamma$-dose, the band related to stretching vibration $\nu_{1}$ of $\mathrm{PO}_{4}^{3-}$ tetrahedral group is presented in Fig. 3. Furthermore, in the part of Raman spectra related 
to the mineral phase of the bone, it is evident, that almost no changes caused by irradiation were observed. Only weak changes were noticed for the dose of 1 MGy. Figure 4 presents the dose dependent Raman band positions of $\delta \mathrm{CH}_{2}$ and $\mathrm{CH}_{2}$ wagging vibrations, which belongs to organic matrix of the bone. It is clearly seen from the Fig. 4, that the frequency of vibration of presented groups undergoes significant changes above $100 \mathrm{kGy}$. Therefore, one can conclude that doses higher than $100 \mathrm{kGy}$ caused great changes in the organic part of the bone.

\subsection{Raman studies of vein walls normal and varicose}

Studies on varicose versus normal veins generally are carried out by histological, microscopic and ultrasonographic methods, which permit to obtain morphological changes. The Raman spectroscopy allows us to search the origin of such morphological changes on the molecular level.

The main molecular constituents of these tissues determine the most important features in the Raman spectra of both type of vein, applied in CABG and vein vessels from chronic venous insufficiency patients. Raman spectra of both type of veins are presented in Fig. 5 . The major bands in recorded spectra are associated with the vibrational modes of collagen and elastin. Results of our preliminary studies revealed the changes in bands related to the vibrations $\nu(\mathrm{C}-\mathrm{C})$ of phospholipids at $\approx 1065 \mathrm{~cm}^{-1}$ and $\approx 1097 \mathrm{~cm}^{-1}$.

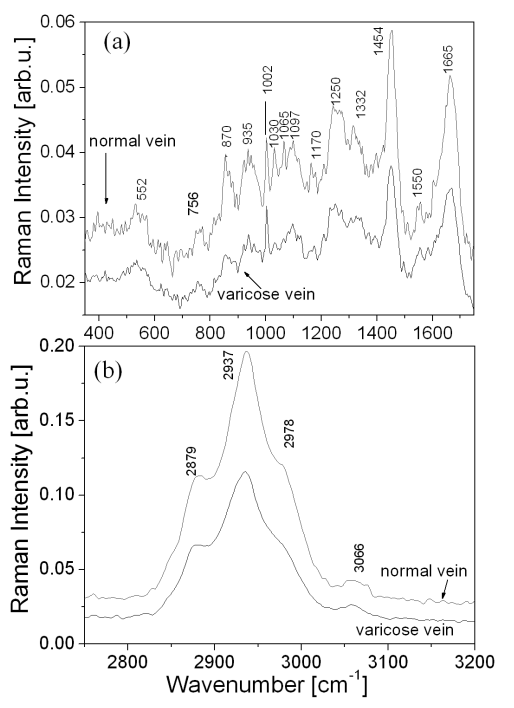

Fig. 5. Raman spectra of normal and varicose veins.

It was also found, that the intensity of the band at $1030 \mathrm{~cm}^{-1}$ (the stretching vibration of the pyridine aromatic ring) decreases significantly in pathological veins. Significant decrease in the intensity of the Raman band related to the symmetric stretching vibration of $\mathrm{CH}_{3}$ entity at $\approx 2936 \mathrm{~cm}^{-1}$ is also observed. The ratio of the band intensity of $\nu\left(\mathrm{CH}_{3}\right)$ to the band intensity of amide I at $\approx 1665 \mathrm{~cm}^{-1}$ decreases from 5.31 for the normal vein to 3.38 for the pathological one.

\subsection{Temperature Raman studies of collagen fibres}

Temperature is one of the most prominent physical factor, which is able to change the protein structure. Heating of collagen leads not only to denaturation but to evacuation of free and bound water as well. The latter process is observed in temperatures below $110^{\circ} \mathrm{C}[13,14]$. The results on temperature effect on Raman spectra of collagen were studied in temperature range from $25^{\circ} \mathrm{C}$ to $110^{\circ} \mathrm{C}$. In Fig. 6. Raman spectra recorded at $25^{\circ} \mathrm{C}$ and $110^{\circ} \mathrm{C}$ are shown. The most prominent temperature changes were observed for the band at $\approx 937 \mathrm{~cm}^{-1}$ assigned to non-aromatic stretching vibration $\nu(\mathrm{C}-\mathrm{C})$. Figure 7 presents the temperature dependence of frequencies of this vibration and the temperature dependence of the vibration $\nu(\mathrm{C}-\mathrm{C})$, which is related to the proline ring. The position and the intensity of the band at $\approx 937 \mathrm{~cm}^{-1}$ decreases with the increasing temperature and, in the vicinity of $80^{\circ} \mathrm{C}$, merge practically with the band at $\approx 922 \mathrm{~cm}^{-1}$. The latter band is related to the vibration of proline ring. The temperature studies carried out on the solid-state collagen revealed, that collagen heated up to $110^{\circ} \mathrm{C}$ is stable, e.g. after cooling to room temperature, the Raman spectrum returned to the original shape. All temperature changes were reversible even for material exposed to several heating-cooling cycles.

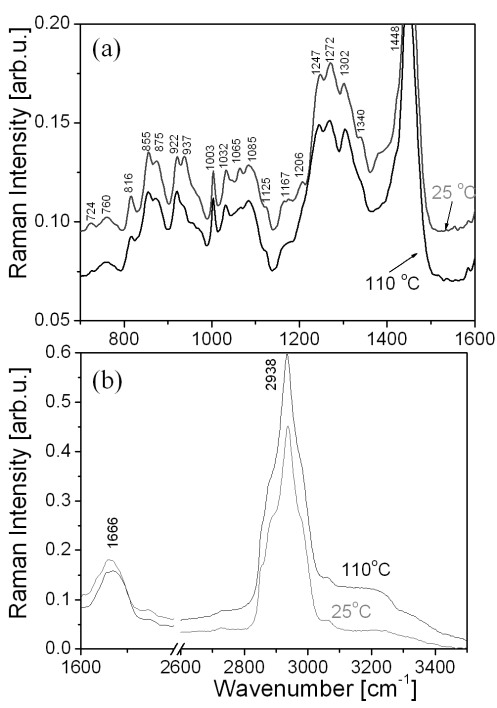

Fig. 6. Raman spectra of collagen at $25^{\circ} \mathrm{C}$ and $110^{\circ} \mathrm{C}$.

\section{Conclusions}

Raman spectroscopy is the useful method in studies on biological materials. It permits the examination of biopolymers such as collagen and even more complex objects as bone and blood vessels. Collected spectra of studied materials exposed to temperature, ionizing radiation and pathological factors converting normal vein into varicose vein, allow us to come to the conclusion on 


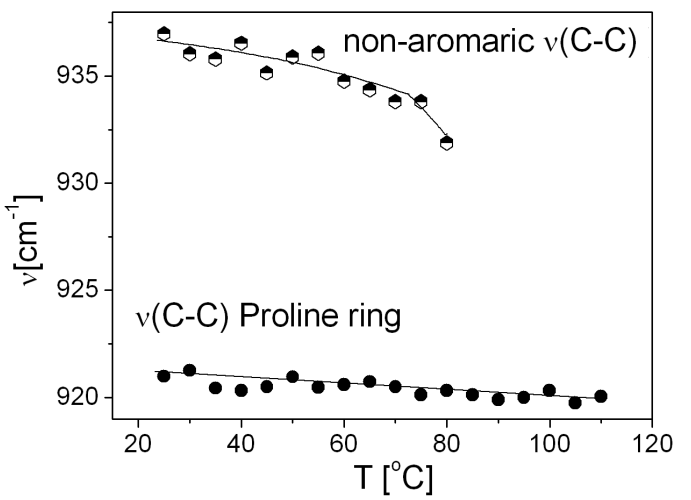

Fig. 7. Temperature dependencies of frequencies of non-aromatic $\nu(\mathrm{C}-\mathrm{C})$ vibration and $\nu(\mathrm{C}-\mathrm{C})$ proline ring vibration of collagen fibres.

the material's susceptibility to the acting factor, thermal stability and reversibility of the process.

\section{References}

[1] J.V. Psala, J. Melhuish, Br. J. Surg. 76, 37 (1989).

[2] M.A. Elsharawy, M.M. Naim, E.M. Abdelmaguid, A.A. Al-Mulhim, Interactive Cardiovascular and Thoracic Surgery 6, 219 (2007).
[3] C. Jeanneret, T. Baldi, S. Hailemariam, C. Koella, J. Gewaltig, B.C. Biedermann, Br. J. Surg. 94, 449 (2007).

[4] B. Schrader, B. Dippel, I. Erb, S. Keller, T. Löchte, T. Schultz, E. Tatsch, S. Wessel, J. Mol. Struct. 480-481, 21 (1999).

[5] B. Schrader, H. Schultz, G.N. Andreev, H.H. Klump, J. Sawatzki, Talanta 53, 35 (2000).

[6] S. Fendel, B. Schrader, Fresenius J. Anal. Chem. 360, 609 (1998).

[7] H.G.M. Edwards, D.W. Farwell, J.M. Holder, E.E. Lawson, J. Mol. Struct. 435, 49 (1997).

[8] A. Carden, M.D. Morris, J. Biomed. Opt. 5, 259 (2000).

[9] G. Frushour, J. Koenig, Biopolymers 14, 379 (1975).

[10] J. Bandekar, Biochim. Biophys. Acta 1120, 123 (1992).

[11] M. Sintzel, A. Merkli, C. Tabatabay, R. Gurny, Drug Development $\&$ Industrial Pharmacy 23, 9 (1997).

[12] L. Kubisz, M. Połomska, Spectrochim. Acta A 66 , 616 (2007).

[13] S. Nomura, A. Hiltner, J.B. Lando, E. Baer, Biopolymers 16, 231 (1977).

[14] L. Kubisz, S. Mielcarek, F. Jaroszyk, Int. J. Biol. Macromol. 33, 1 (2003). 